Fundación

Miguel Lillo

Tucumán

Argentina

\title{
Nuevos registros de Algas verdes (Chlorophyta) de ríos y arroyos serranos de la provincia de Córdoba, Argentina
}

\author{
New records of Green algae (Chlorophyta) in mountain rivers and \\ streams in the province of Córdoba, Argentina
}

\footnotetext{
Daga, I. Claudia ${ }^{1 *}$ (1); M. Cecilia Fernández Belmonte ${ }^{2}$ (1)

1 Cátedra de Diversidad Biológica I, Facultad de Ciencias Exactas Físicas y Naturales, Universidad Nacional de Córdoba. Avenida Vélez Sarsfield 299, Córdoba, Argentina.

2 Cátedra de Morfología Vegetal, Facultad de Ingeniería y Ciencias Agropecuarias, Universidad Nacional de San Luis. Ruta 148 Ext. Norte, Villa Mercedes, San Luis, Argentina.

* Autor corresponsal: inesclaudiadaga@gmail.com
}

\section{RESUMEN}

El objetivo de este trabajo fue ampliar el conocimiento de las algas verdes de los tributarios del embalse San Roque (ríos San Antonio y Cosquín, arroyos Las Mojarras y Los Chorrillos) y el embalse La Quebrada (arroyos Colanchanga y Los Hornillos). Las muestras fueron recolectadas estacionalmente durante los años 2014 a 2016. Se identificaron un total de 66 taxones: Chlorophyceae (39), Zygnematophyceae (22), Trebouxiophyceae (3) y Ulvophyceae (2). La mayor riqueza de especies ocurrió en primavera y el río San Antonio fue el tributario que presentó mayor número de taxones. Se mencionan especies indicadoras de eutrofia como Monoraphidium griffithii; Actinastrum hantzschii, Monactinus simplex, Nephrocytium agardhianum, Scenedesmus subspicatus, Staurastrum leptocladum, Closterium aciculare y a Phacotus lenticularis, especie indicadora de ambientes con carbonato de calcio.

Palabras clave - Algas bioindicadoras; clorofitas; organismos planctónicos.

\begin{abstract}
The objective of this work was to expand the knowledge on green algae of the tributaries of San Roque Reservoir (San Antonio and Cosquín rivers, Las Mojarras and Los Chorrillos streams) and La Quebrada Reservoir (Colanchanga and Los Hornil-

- Ref. bibliográfica: Daga, I. C.; Fernández Belmonte, M. Cecilia. 2021. Nuevos registros de Algas verdes (Chlorophyta) de ríos y arroyos serranos de la provincia de Córdoba, Argentina. Lilloa 58 (2): 95-114. doi: https://doi.org/10.30550/j.lil/2021.58.2/2021.10.19

> Recibido: 23 de agosto 2021 - Aceptado: 19 de octubre 2021 - Publicado en línea: ... de 2021.

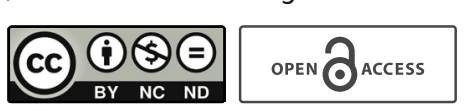

> URL de la revista: http://lilloa.lillo.org.ar

> Esta obra está bajo una Licencia Creative Commons Atribución - No Comercial - Sin Obra Derivada 4.0 Internacional.
\end{abstract}


los streams). The samples were collected seasonally from 2014 to 2016 . A total of 66 taxa were identified: Chlorophyceae (39), Zygnematophyceae (22), Trebouxiophyceae (3) and Ulvophyceae (2). The highest species richness occurred in spring and tributary with the highest number of taxa was San Antonio River. The following eutrophication indicator species mentioned are: Actinastrum hantzschii, Monactinus simplex, Nephrocytium agardhianum, Scenedesmus subspicatus, Staurastrum leptocladum, Closterium aciculare and Phacotus lenticularis, an indicator species of environments with calcium carbonate.

Keywords - Bioindicators algae; Chlorophytes; planktonic organisms.

\section{INTRODUCCIÓN}

La provincia de Córdoba presenta una amplia red hidrográfica integrada en su mayor parte por ríos y arroyos que nacen en las serranías, se pueden diferenciar las nacientes de los cursos de agua con lechos rocosos, saltos y rápidos mientras que en las desembocaduras a lagos y embalses presentan sectores areno-gravosos. Son ambientes altamente diversos donde la combinación de las variables geomorfológicas, hidrológicas y bióticas inciden sobre el establecimiento y permanencia de las comunidades algales (Luque y Martínez de Fabricius, 2005; Galea et al., 2014; Bojorge-García y Cantoral Uriza, 2016). Estas comunidades son consideradas los productores primarios de mayor relevancia, se encuentran en la base de la cadena alimentaria y juegan un papel importante en los procesos de circulación de la materia y transferencia de energía (Harris, 1986; Salmaso et al., 2015). Otro aspecto interesante de las algas es que pueden ser utilizadas como indicadoras de calidad de agua debido a su pronta respuesta a los impactos antrópicos, como exceso de nutrientes y sustancias tóxicas (Reynolds et al., 2002; Brettum y Andersen, 2005).

Estudios realizados en ríos y arroyos del país y otras latitudes señalan que las algas verdes luego de las diatomeas son las que efectúan el mayor aporte en riqueza de especies, así lo indican trabajos que se llevaron a cabo en cuerpos de agua del sur de la provincia de Córdoba (Luque y Martínez de Fabricius, 2000, 2005). Resultados similares se obtuvieron en otros ríos y arroyos del país (Moraña, 2013; Taboada y Tracanna 2013; Galea et al., 2014) y para el hemisferio norte (Esho y Benson-Evans, 1984; Yulu et al., 2021).

El objetivo del presente trabajo fue determinar taxonómicamente las algas verdes de los arroyos y ríos serranos tributarios de los embalses San Roque y La Quebrada, analizar la variabilidad estacional, su abundancia e indicar aquellas especies que revistan interés como potenciales bioindicadoras ambientales.

\section{MATERIALES Y MÉTODOS}

El sistema de arroyos y ríos estudiados están comprendidos aproximadamente entre los meridianos $64^{\circ} 20^{\prime}$ y $64^{\circ} 29^{\prime}$ de longitud Oeste y los paralelos $31^{\circ} 08^{\prime}$ y $31^{\circ} 24^{\prime}$ de 
latitud Sur, pertenecen a la cuenca alta del Río Suquía (Primero) que tiene como nivel de base la Laguna de Mar Chiquita.

El clima en la zona es templado, la temperatura media anual es de $18,9^{\circ} \mathrm{C}$ (Capitanelli, 1979). La cuenca está caracterizada por una precipitación media anual de 780 mm (Colladón, 2018). La mayor parte de las precipitaciones ocurren entre octubre y abril, los cursos de agua tienen un comportamiento que acompaña el régimen de lluvias, presentando niveles mínimos al final del invierno y valores máximos en época estival. En la actualidad se observa una alternancia de años muy húmedos y otros secos con escenarios hidrológicos contrastantes de sequías e inundaciones extremas (Dasso et al., 2014). Tal situación se vio reflejada en el verano de 2015 donde las abundantes precipitaciones caídas en las sierras chicas ocasionaron inundaciones que imposibilitaron la toma de muestras durante ese verano.

El muestreo se realizó estacionalmente entre los años 2014 a 2016 en las desembocaduras de los tributarios del embalse San Roque: Río Cosquín, Arroyo Las Mojarras, Arroyo Los Chorrillos, Río San Antonio y los tributarios del embalse La Quebrada: Arroyo Colanchanga y Arroyo Los Hornillos (Fig. 1). Para la recolección

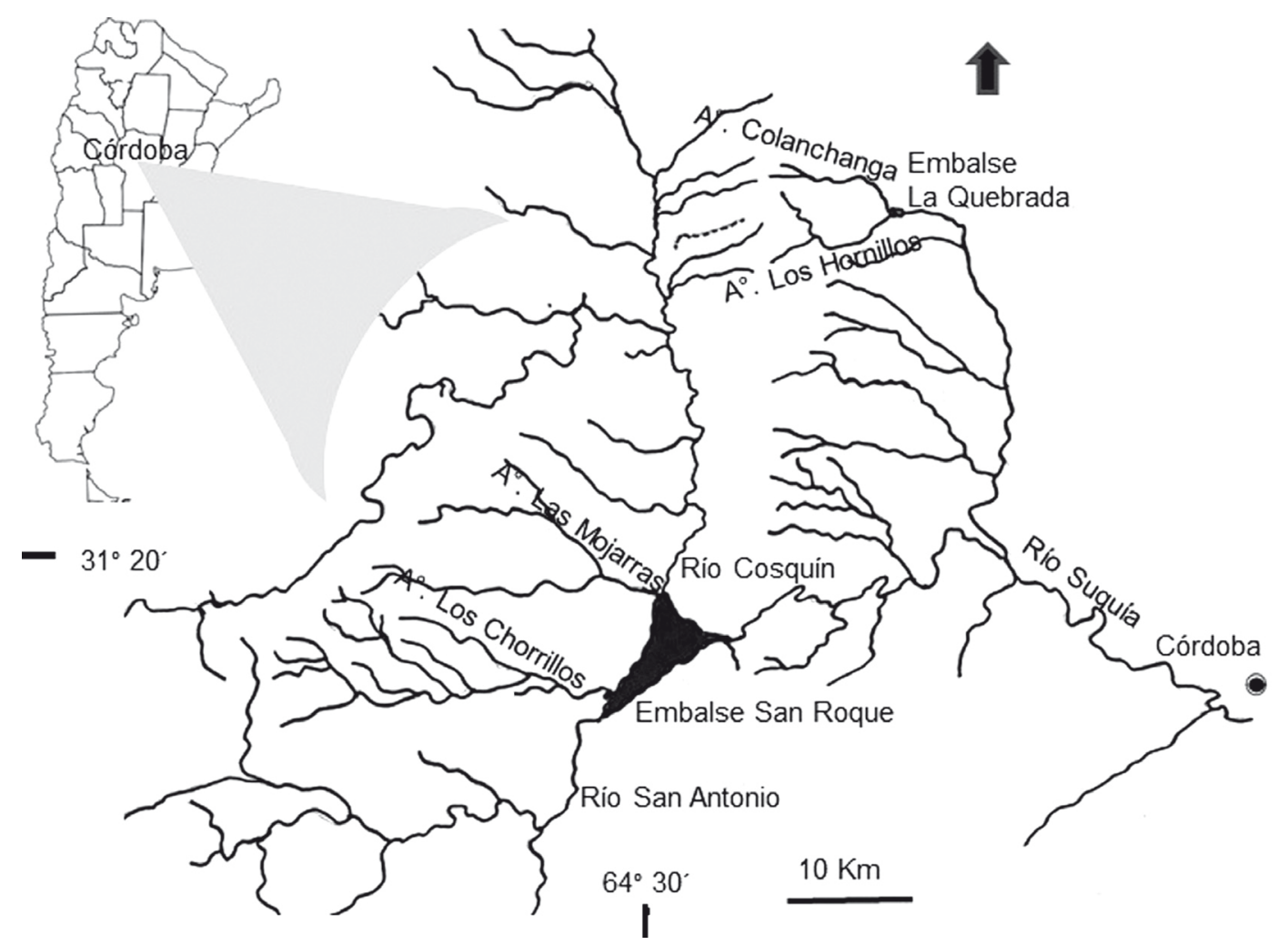

Fig. 1. Cuenca hidrográfica del río Suquía (modificado de Vázquez et al., 1979). Referencias- Tribu-

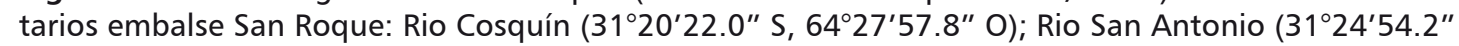

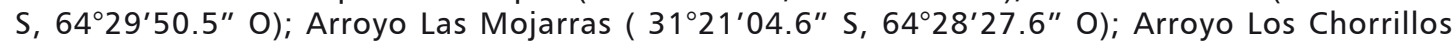
$\left(31^{\circ} 24^{\prime} 03.7^{\prime \prime}\right.$ S, 6429'46.7" O). Embalse La Quebrada: Arroyo Colanchanga (310.'58.62" S,

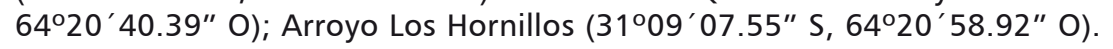

Fig. 1. Hydrographic basin of the Suquía river (modified from Vázquez et al., 1979). ReferencesSan Roque Reservoir tributaries: Cosquin river $\left(31^{\circ} 20^{\prime} 22.0\right.$ "S, 64 $\left.27^{\prime} 57.8^{\prime \prime} \mathrm{W}\right)$; San Antonio river

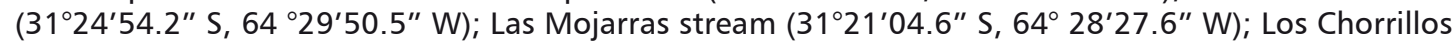

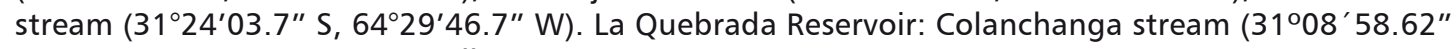

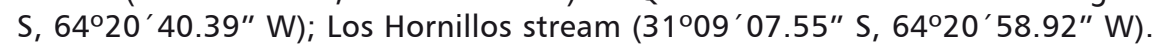


se utilizó una red de plancton de $20 \mu \mathrm{m}$ de diámetro de poro, filtrando un volumen de agua de unos 10 litros a lo largo de una transecta perpendicular al cauce. Ese filtrado se introdujo en una botella plástica y se conservó a oscuras para su posterior determinación. Con instrumentos portátiles se hicieron mediciones in situ de la temperatura y $\mathrm{pH}$ (pHTestr 30), conductividad eléctrica (ECTestr 11) y oxígeno disuelto (Acorn DO 6). El análisis taxonómico se realizó mediante el empleo de microscopio óptico marca Kyowa (mod. LSCB-VC-2B-L). Las muestras fueron observadas en vivo a 10, 40 y 100x. Los materiales fueron dibujados y fotografiados con cámara digital, luego fueron etiquetados e incorporaron al herbario del Área de Proyectos Especiales de la Facultad de Ciencias Exactas Físicas y Naturales de la Universidad Nacional de Córdoba (PE. FCEFyN-UNC).

En las determinaciones taxonómicas se utilizó la siguiente bibliografía: Tell (1979); Ettl (1981); Komarek y Fott (1983), Huber-Pestalozzi (1961) y Prescott (1982). Se consultó la base de datos taxonómica para algas y cianobacterias AlgaeBase, accesible en internet (Guiry y Guiry, 2021). Para la distribución geográfica de las especies se utilizó el catálogo de Tell (1985). Las muestras se conservaron en heladera $\left(4-10^{\circ} \mathrm{C}\right)$ para su posterior observación e identificación in vivo. Una parte de la muestra se fijó con Lugol (0,5 a $1 \mathrm{~mL}$ por cada $100 \mathrm{~mL}$ de muestra) para realizar los recuentos y otras en formaldehído (2-4 \%) para su conservación a largo plazo. Fueron etiquetadas y se incorporaron al herbario del Área de Proyectos Especiales de la Facultad de Ciencias Exactas Físicas y Naturales de la Universidad Nacional de Córdoba.

El recuento de células se realizó utilizando una cámara de Sedgwick-Rafter y un microscopio óptico con un ocular de 15 aumentos y un objetivo de 10x. Primero se procedió a homogeneizar la muestra, luego se llenó la cámara con una pipeta graduada y se dejó reposar unos 15 minutos. El recuento fue generalmente de toda la cámara, excepcionalmente cuando la muestra fue muy densa se contaron un número determinado de transectas, los valores que se obtuvieron se expresaron en células $\mathrm{mL}^{-1}$.

Se confeccionó un listado de las especies identificadas en cada uno de los sitios de muestreo (se detallan en la Tabla 2) y se realizó una breve descripción de los nuevos registros para la provincia de Córdoba de acuerdo con bibliografía consultada.

\section{RESULTADOS}

Los valores medios de las variables físicas y químicas de los sitios de muestreo se encuentran en la Tabla 1.

La temperatura del agua presentó una media anual entre 20 y $24^{\circ} \mathrm{C}$. En el arroyo Colanchanga se observaron las temperaturas más bajas, situación que puede ser atribuida a la abundante vegetación de ribera a lo largo del cauce que disminuye la intensidad lumínica y con ello la temperatura.

Los ambientes estudiados se caracterizaron por presentar aguas alcalinas con valores medios de $\mathrm{pH}$ entre 8,6-9,0.

La conductividad presentó valores medios entre 227 y $480 \mu \mathrm{S} \mathrm{cm}^{-1}$. Los registros mayores corresponden al río Cosquín y arroyo Colanchanga. 
Tabla 1. Valores medios de las variables físicas y químicas de los sitios de muestreo.

Table 1. Average values of the physical and chemical variables of the sampling sites.

\begin{tabular}{|l|c|c|c|c|}
\multirow{2}{*}{ Sitios de muestreo } & \multicolumn{4}{|c}{ Parámetros } \\
\cline { 2 - 5 } & Temp. ${ }^{\circ} \mathbf{C}$ & $\mathrm{pH}$ & Cond. $\left(\boldsymbol{\mu S} \mathrm{cm}^{-1}\right)$ & OD $\left(\mathrm{mg} \mathrm{L}^{-1}\right)$ \\
\hline Río Cosquín & $22,85 \pm 5,48$ & $8,66 \pm 0,27$ & $480,38 \pm 141,7$ & $6,53 \pm 2,16$ \\
\hline Arroyo Las Mojarras & $23,2 \pm 5,42$ & $8,65 \pm 0,32$ & $275,25 \pm 78,98$ & $6,72 \pm 2,36$ \\
\hline Arroyo Los Chorrillos & $24,03 \pm 5,7$ & $9,05 \pm 0,26$ & $227,75 \pm 23,86$ & $6,55 \pm 3,12$ \\
\hline Río San Antonio & $23,48 \pm 5,6$ & $8,68 \pm 0,13$ & $314,63 \pm 92,76$ & $6,64 \pm 2,25$ \\
\hline Arroyo Colanchanga & $20,24 \pm 5,23$ & $8,56 \pm 0,25$ & $348,5 \pm 39,13$ & $8,64 \pm 1,98$ \\
\hline Arroyo Los Hornillos & $22,79 \pm 5,63$ & $8,88 \pm 0,37$ & $258,27 \pm 104,64$ & $8,22 \pm 1,64$ \\
\hline
\end{tabular}

La concentración de oxígeno disuelto fluctuó entre 6,53 y 8,64 $\mathrm{mg} \mathrm{L}^{-1}$. En otoñoinvierno se observaron los registros mayores y en primavera-verano los menores.

Se determinaron un total de 66 taxones, 39 pertenecen al grupo de las Chlorophyceae, 22 a Zygnematophyceae, 3 a Trebouxiophyceae y 2 Ulvophyceae (Tabla 2). Los géneros Scenedesmus y Closterium aportaron el mayor número de taxones. Scenedesmus dimorphus, Selenastrum gracile y Closterium aciculare se observaron en todos los sistemas lóticos a excepción del arroyo Los Chorrillos.

La distribución espacial fue muy heterogénea, en el río San Antonio se identificaron 51 taxones y en el resto de los tributarios el número de especies osciló entre 6 y 16.

La primavera fue la estación del año donde fueron más representativos los grupos estudiados a excepción de las Ulvophyceae: Cladophora glomerata y Chaetomorpha herbipolensis que se registraron durante el invierno.

En las Figs. 2 y 3 se observan las variaciones estacionales de la densidad de algas verdes (células $\mathrm{mL}^{-1}$ ). En el primer año de estudio la mayor densidad celular ocurrió en la primavera con 84 células $\mathrm{mL}^{-1}$ en el río San Antonio y 120 células $\mathrm{mL}^{-1}$ en el arroyo Colanchanga. El mayor aporte corresponde a organismos cenobiales como Scenedesmus, Coelastrum, Pediastrum y Desmodesmus para el río San Antonio y a Phacotus lenticularis para el arroyo Colanchanga (Fig. 2). En el segundo año de estudio la máxima densidad ocurrió en el rio Cosquín con 396 células $\mathrm{mL}^{-1}$ durante el verano de 2016, el organismo dominante fue P. lenticularis. El arroyo Los Chorrillos presentó la menor densidad de algas verdes en ambos períodos estudiados coincidiendo también con la menor riqueza de especies (Fig. 3).

A continuación, se realiza una breve descripción de las especies reportadas por primera vez para la provincia de Córdoba.

\section{División ChLOROPHYTA \\ Clase TREBOUXIOPHYCEAE \\ Pachycladella umbrina (G.M. Smith) P. C. Silva 1970 (Fig. 4I)}

Células esféricas con un cloroplasto y un pirenoide, pared celular prolongada en cuatro procesos huecos dispuestos en forma tetraédrica con extremos bifurcados.

- Diámetro celular 8-12 $\mu \mathrm{m}$; proyecciones largo $28-40 \mu \mathrm{m}$. 


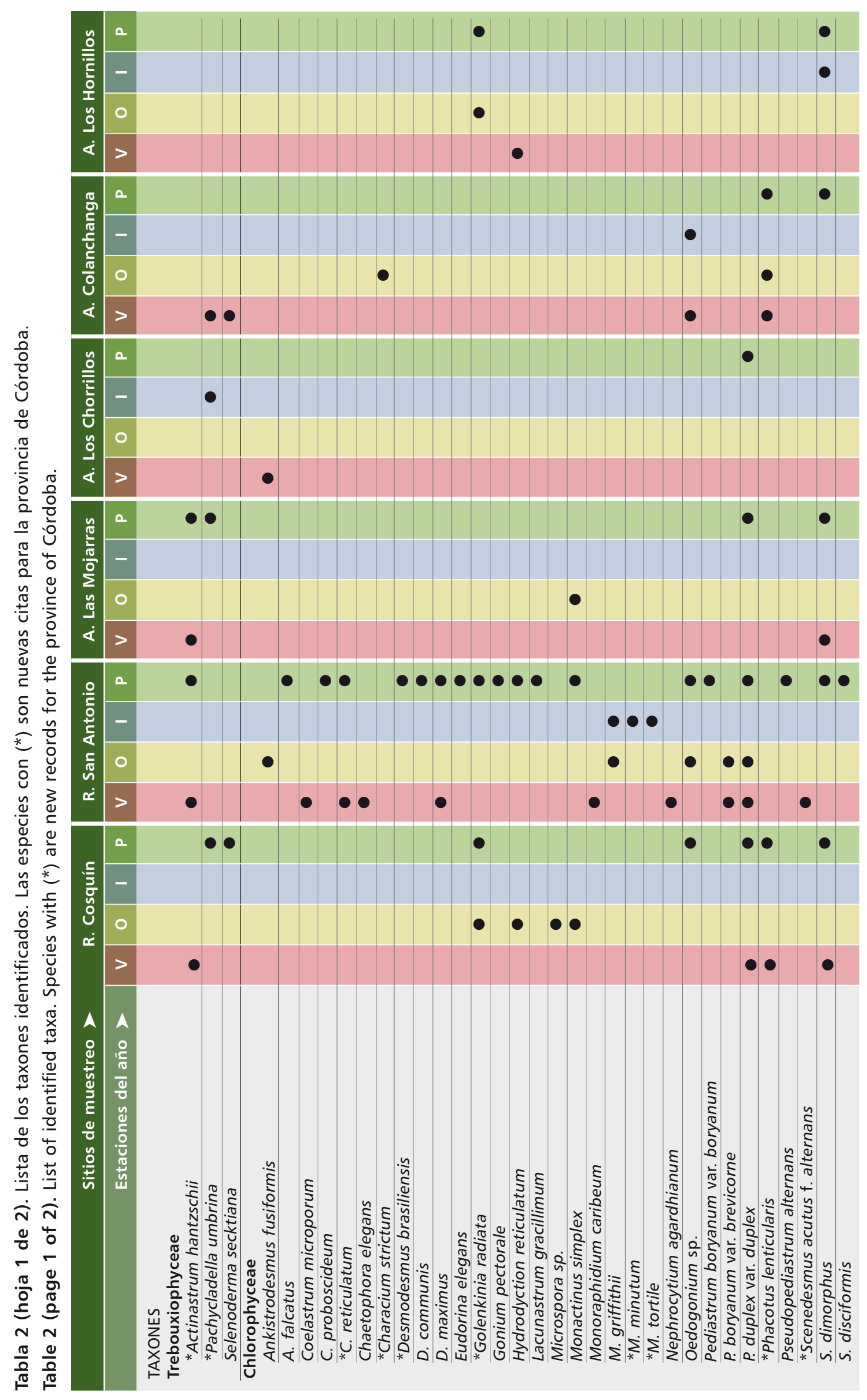




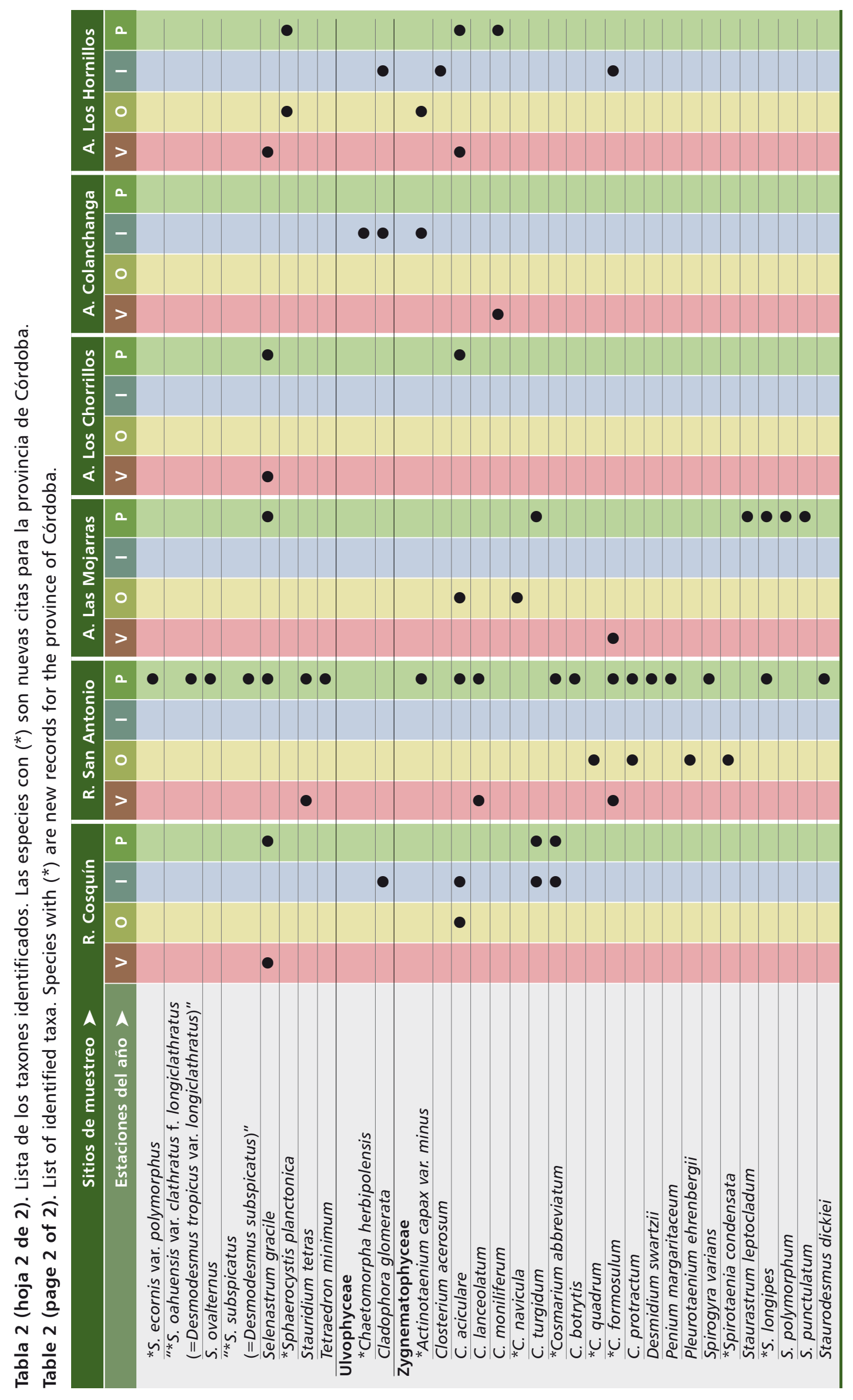




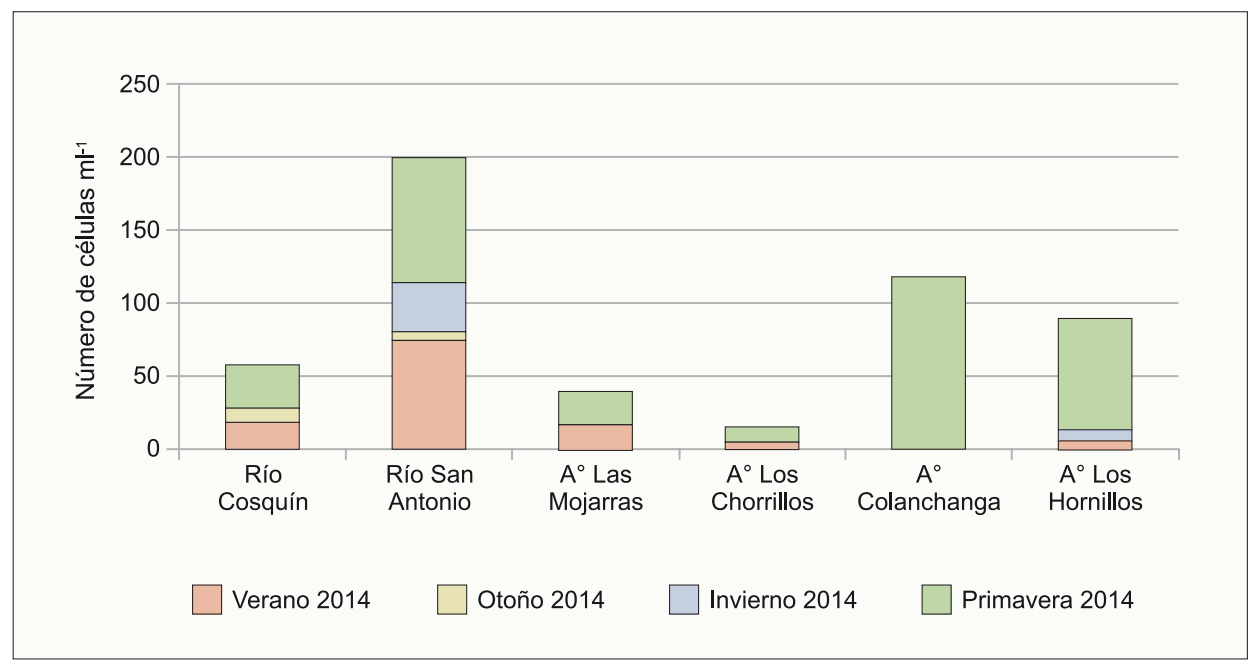

Fig. 2. Variación estacional de la densidad de algas verdes (células $\mathrm{ml}^{-1}$ ) en los ríos y arroyos estudiados (período: 2014).

Fig. 2. Seasonal variation in the density of green algae (cells $\mathrm{ml}^{-1}$ ) in the studied rivers and streams (period: 2014).

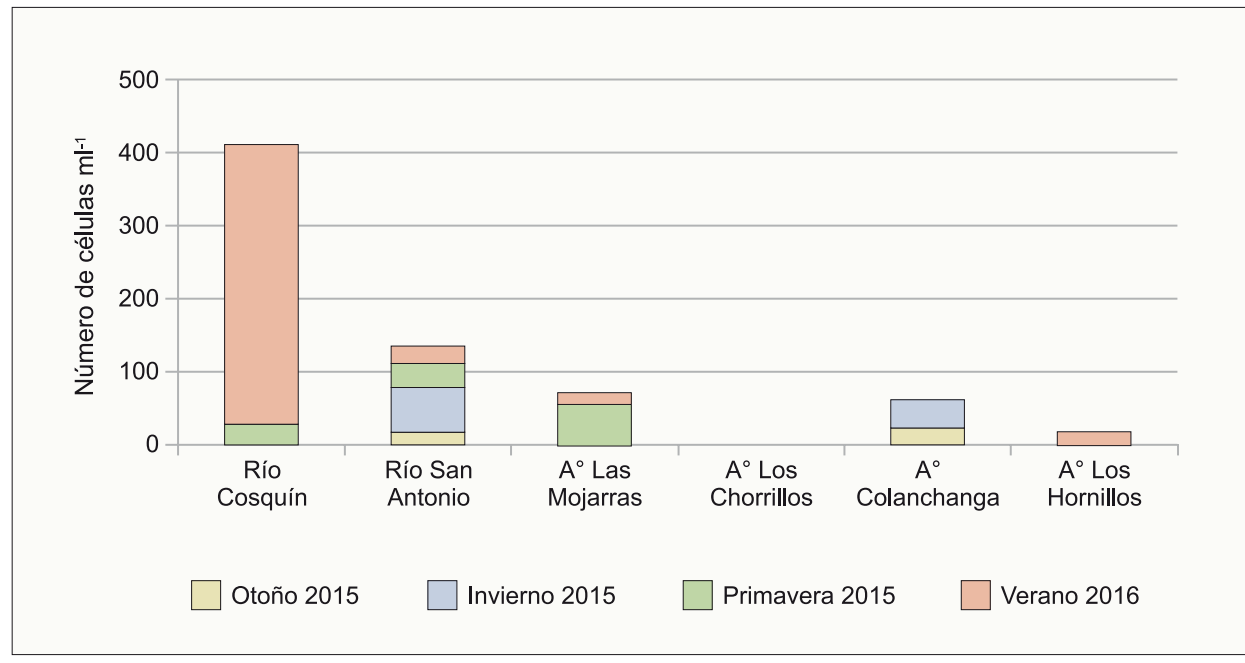

Fig. 3. Variación estacional de la densidad de algas verdes (células $\mathrm{ml}^{-1}$ ) en los ríos y arroyos estudiados (período: otoño 2015-verano 2016).

Fig. 3. Seasonal variation in the density of green algae ( $\mathrm{ml}^{-1}$ cells) in the studied rivers and streams (period: Autumn 2015- Summer 2016).

- Hábitat y distribución: planctónica de aparición esporádica. En Argentina citada para el Río Paraná y Paraguay (Zalocar de Domitrovic, 1999).

- Material examinado: Argentina. Prov. Córdoba, Dptos. Punilla y Colón (arroyos Los Chorrillos, Las Mojarras y río Cosquín), 2014 a 2016, Daga I.C. 147a, 147b, 147c, 194 (PE. FCEFyN-UNC). 


\section{Clase CHLOROPHYCEAE Actinastrum hantzschii Lagerheim 1882 (Fig. 4A)}

Cenobios de 2 a 8 células que irradian desde un centro común, las células tienen forma de clava, con un cloroplasto y un pirenoide. Diámetro del cenobio $25 \mu \mathrm{m}$; ancho de la célula de 2,5-3 $\mu \mathrm{m}$; largo de 10-12 $\mu \mathrm{m}$.

- Hábitat y distribución: planctónica en ambientes mesotróficos a eutróficos (Pinilla, 2000). En Argentina citada para Buenos Aires, Corrientes, Santa Fe (Tell, 1985); La Pampa (Bazán et al., 2014).

- Material examinado: Argentina. Prov. Córdoba, Dpto. Punilla (rio Cosquín, San Antonio, arroyo Las Mojarras), 2014 a 2016, Daga I.C. 140a, 141b, 189 (PE. FCEFyN-UNC).

\section{Characium strictum A. Braun 1855 (Fig. 4B y 4C)}

Células solitarias, ovoides a elipsoidales, con un pedículo corto y un disco de fijación, cloroplasto parietal con un pirenoide. Largo de la célula $16-20 \mu \mathrm{m}$; ancho de 6-8 $\mu \mathrm{m}$.

- Hábitat y distribución: epifita, sobre Chaetomorpha herbipolensis. En Argentina citada para Buenos Aires (Tell, 1985).

- Material examinado: Argentina. Prov. Córdoba, Dpto. Colón (arroyo Colanchanga), 2014 a 2016, Daga I.C. 160a (PE. FCEFyN-UNC).

\section{Coelastrum reticulatum (Dangeard) Senn 1899 (Fig. 4D)}

Cenobios esféricos a elipsoidales formados por 8, 16 hasta 32 células esféricas provistos de una envoltura mucilaginosa. A menudo los cenobios son múltiples conectados entre sí por proyecciones cilíndricas dando el aspecto de un retículo. Diámetro del cenobio $40 \mu \mathrm{m}$; diámetro celular de 2-6 $\mu \mathrm{m}$.

- Hábitat y distribución: planctónica en ríos y lagos poco profundo y con nutrientes (Reynold et al., 2002). En Argentina citada para Corrientes (Tell, 1985); Río Paraná (Zalocar de Domitrovic, 1999).

- Material examinado: Argentina. Prov. Córdoba, Dpto. Punilla (río San Antonio), 2014 a 2016, Daga I.C. 156, 189 (PE. FCEFyN-UNC).

\section{Desmodesmus brasiliensis (Bohlin) Hegewald 2000 (Fig. 4E)}

Cenobios planos, lineales, formados por 2 a 4 células, elípticas a oblongas de polos redondeados. Con 1 a 3 espinas que pueden estar presentes en todas las células del cenobio. Presentan una costilla longitudinal mediana a lo largo de toda la célula. Cloroplasto parietal con un pirenoide. Células con largo de 10-16 $\mu \mathrm{m}$; ancho de 3,5-5 $\mu \mathrm{m}$; espinas: $1-2 \mu \mathrm{m}$.

- Hábitat y distribución: planctónica en aguas mesotróficas a eutróficas (Novelo, 2012). Sin datos en Argentina (Tell, 1985). 

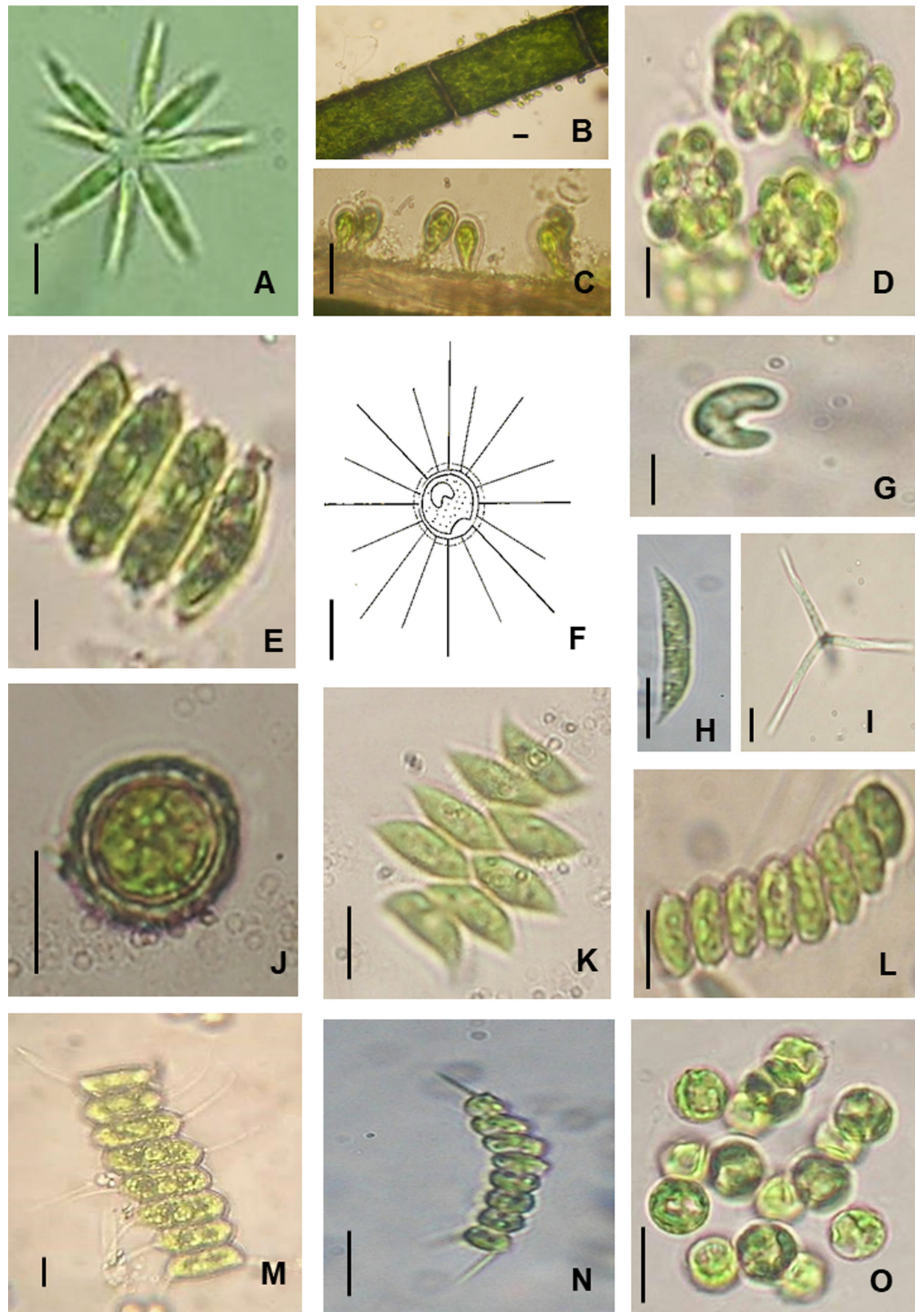

Fig. 4. A) Actinastrum hantzschii. B-C) Characium strictum. D) Coelastrum reticulatum. E) Desmodesmus brasiliensis. F) Golenkinia radiata. G) Monoraphidium minutum. H) M. tortile. I) Pachycladella umbrina. J) Phacotus lenticularis. K) Scenedesmus ecornis var. polymorphus. L) S. acutus f. alternans. M) S. oahuensis f. longiclathratus (= Desmodesmus tropicus var. longiclathratus). N) S. subspicatus (=Desmodesmus subspicatus). O) Sphaerocystis planctonica. Escala $=10 \mu \mathrm{m}$

Fig. 4. A) Actinastrum hantzschii. B-C) Characium strictum. D) Coelastrum reticulatum. E) Desmodesmus brasiliensis. F) Golenkinia radiata. G) Monoraphidium minutum. H) M. tortile. I) Pachycladella umbrina. J) Phacotus lenticularis. K) Scenedesmus ecornis var. polymorphus. L) S. acutus f. alternans. M) S. oahuensis f. longiclathratus (= Desmodesmus tropicus var. longiclathratus). N) S. subspicatus (=Desmodesmus subspicatus). O) Sphaerocystis planctonica. Scale $=10 \mu \mathrm{m}$ 
- Material examinado: Argentina. Prov. Córdoba, Dpto. Punilla (río San Antonio), 2014 a 2016, Daga I.C. 156 (PE. FCEFyN-UNC).

\section{Golenkinia radiata Chodat 1894 (Fig. 4F)}

Células esféricas, solitarias con setas largas delicadas hialinas. Cloroplasto en forma de copa con un pirenoide. Cuerpo celular con diámetro de 10-15 $\mu \mathrm{m}$; setas largo de $22 \mu \mathrm{m}$.

- Hábitat y distribución: planctónica en cuerpos de agua pequeños, probablemente cosmopolita, aunque no muy frecuente (Komárek y Fott, 1983). En Argentina citada para Buenos Aires, Santa Fe y Tierra del Fuego (Tell, 1985).

- Material examinado: Argentina. Prov. Córdoba, Dptos. Punilla y Colón (río San Antonio y arroyo Los Hornillos), 2014 a 2016, Daga I.C. 158, 160a, 160b, 175 (PE. FCEFyN-UNC).

\section{Monoraphidium minutum (Nägeli) \\ Komárková-Legnerová 1969 (Fig. 4G)}

Células solitarias en forma de media luna, los extremos celulares a veces se acercan entre sí, algo atenuados y redondeados. Cloroplasto parietal sin pirenoide. Células: largo de 6-12 $\mu \mathrm{m}$; ancho de 2-4 $\mu \mathrm{m}$.

- Hábitat y distribución: planctónica, cosmopolita frecuentemente en ambientes tropicales y de aguas alcalinas (Comas 1996). En Argentina citada para Santa Fe (Tell, 1985).

- Material examinado: Argentina. Prov. Córdoba, Dpto. Punilla (río San Antonio), 2014 a 2016, Daga I.C. 175 (PE. FCEFyN-UNC).

\section{Monoraphidium tortile (West G. S. West) \\ Komárková-Legnerová 1969 (Fig. 4H)}

Células solitarias, en forma de huso, recta, curva, sigmoidal, o retorcida en espiral, sin vaina de mucílago. Ambos extremos de la célula de manera similar, curvos. Cloroplasto único y parietal, sin pirenoide. Células: largo de 30-35 $\mu \mathrm{m}$; ancho de 3-3,5 $\mu \mathrm{m}$.

- Hábitat y distribución: planctónica y metafítica en cuerpos de agua mesotróficos y eutróficos (Komárek y Fott, 1983). En Argentina citada para Tierra del Fuego (Tell, 1985).

- Material estudiado: Argentina. Prov. Córdoba, Dpto. Punilla (río San Antonio), 2014-2016, Daga I. C. 175 (PE. FCEFyN-UNC). 


\title{
Phacotus lenticularis (Ehrenberg) Diesing 1866 (Fig. 4J)
}

Células solitarias con una lorica formada por dos mitades iguales impregnada de carbonato de calcio. Cloroplasto en forma de copa, pirenoide uno a numerosos. Con dos flagelos idénticos. Célula: largo de 6-8 $\mu \mathrm{m}$; ancho de 5-7 $\mu \mathrm{m}$; diámetro lorica de $12-18 \mu \mathrm{m}$.

- Hábitat y distribución: planctónica, citada para lagos oligotróficos a hipereutróficos y para ríos de pocos movimientos. Es una especie de aparición esporádica, predominante de verano a otoño en aguas alcalinas ricas en carbonato de calcio, se ha visto que el número de individuos aumenta con el estado trófico del cuerpo de agua (Gruenert y Raeder, 2014). Su presencia se relacionó con una disminución de la corriente confiriéndole al agua una coloración blanca lechosa. Citada para Buenos Aires (Tell, 1985).

- Material examinado: Argentina. Prov. Córdoba, Dptos. Punilla y Colón (rio Cosquín y arroyo Colanchanga), 2014-2016, Daga I. C. 158,166, 192 (PE. FCEFyNUNC).

\section{Scenedesmus acutus f. alternans Hortobagyi 1941 (Fig. 4L)}

Cenobio de 4 a 8 células fusiformes dispuestas en un plano o alternadas. Largo de la célula $12 \mu \mathrm{m}$; ancho $4 \mu \mathrm{m}$.

- Hábitat y distribución: planctónica. En Argentina citada para Tierra el Fuego (Tell, 1985).

- Material examinado: Argentina. Prov. Córdoba, Dpto. Punilla (río San Antonio), 2014-2016, Daga I. C. 189 (PE. FCEFyN-UNC).

\section{Scenedesmus ecornis var. polymorphus Chodat 1926 (Fig. 4K)}

Cenobios de 2 a 16 células dispuestos linealmente en zigzag, células elipsoidales u ovoides con extremos redondeados a angulosos. Largo de la célula de 8-12 $\mu \mathrm{m}$; ancho $4-5 \mu \mathrm{m}$.

- Hábitat y distribución: planctónica. Sin datos en Argentina (Tell, 1985).

- Material examinado: Argentina. Prov. Córdoba, Dpto. Punilla (rio Cosquín), 2014-2016, Daga I. C. 156 (PE. FCEFyN-UNC).

\author{
Scenedesmus oahuensis f. longiclathratus G. Tell 1979 \\ (= Desmodesmus tropicus var. longiclathratus (Tell) \\ S. L. Jeon \& E. Hegewal) (Fig. 4M)
}

Cenobio compuesto por 4 a 8 células, cilíndricas a elipsoidales con los extremos cónicos a redondeados dispuestas en líneas. Las espinas de las células pueden ser rectas 0 ligeramente convexas. Largo celular entre $25-30 \mu \mathrm{m}$; ancho 6-8 $\mu \mathrm{m}$; espinas $8 \mu \mathrm{m}$.

- Hábitat y distribución: planctónica. En Argentina citada para Corrientes (Tell, 1979). 
- Material examinado: Argentina. Prov. Córdoba, Dpto. Punilla (río San Antonio), 2014-2016, Daga I. C. 156 (PE. FCEFyN-UNC).

- Observaciones: los organismos identificados presentan células de menor tamaño que las citadas en la bibliografía.

\section{Scenedesmus subspicatus Chodat 1926 (=Desmodesmus subspicatus}

[R. Chodat] Hegewald et A. Schmid) (Fig. 4N)

Cenobios generalmente de 4 células, pero hay de 2 y 8, dispuestos linealmente, a veces ligeramente alternados; células elipsoidales a ampliamente ovoides, ápices redondeados; células marginales llevan una sola espina grande en cada vértice y 1 a 3 espinas adicionales, el resto de las células del cenobio solo llevan una espina. Largo celular 6-8 $\mu \mathrm{m}$; ancho de 4-4,5 $\mu \mathrm{m}$; espinas de $4 \mu \mathrm{m}$

- Hábitat y distribución: planctónica. Citada para ambientes mesotróficos a eutróficos (Phinyo et al., 2017). Sin datos en Argentina (Tell, 1985).

- Material examinado: Argentina. Prov. Córdoba, Dpto. Punilla (río San Antonio), 204-2016, Daga I. C. 188 (PE. FCEFyN-UNC).

\section{Sphaerocystis planctonica (Korshikov) Bourrelly 1974 (Fig. 4O)}

Colonias de 4 a 16 células, dispuestas en un mucílago homogéneo. Células globosas con un cloroplasto en forma de copa y un pirenoide. Diámetro entre 6-20 $\mu \mathrm{m}$.

- Hábitat y distribución: planctónica, indicadora de ambientes oligotróficos (Reynolds et al., 2002). En Argentina citada para río Paraná y el río Paraguay (Zalocar de Domitrovic, 1999).

- Material examinado: Argentina. Prov. Córdoba, Dpto. Colón (arroyo Los Hornillos), 2014-2016, Daga I. C.158b, 166 (PE. FCEFyN-UNC).

\section{Clase UlvophyceAe}

Chaetomorpha herbipolensis Lagerheim 1887 (Fig. 5A y 5B)

Talo formado por filamentos uniseriados, no ramificados que se fijan al sustrato por una célula ensanchada. Pared celular gruesa, cloroplasto reticulado con numerosos pirenoides. Crece formando matas densas de coloración verde oscura. Filamentos hasta $10 \mathrm{~cm}$ de largo; longitud celular de 160-300 $\mu \mathrm{m}$; ancho celular de 90-100 $\mu \mathrm{m}$; pared $6 \mu \mathrm{m}$.

- Hábitat y distribución: perifítica, crece adherida a rocas generalmente, agua de corrientes rápidas de hasta $50 \mathrm{~cm}$ de profundidad. Se la encontró con un gran número de epifitas. Stoyneva y Gärtner (2004) citan a esta especie como planctónica. Sin datos en Argentina (Tell, 1985).

- Material examinado: Argentina. Prov. Córdoba, Dpto. Colón (arroyo Colanchanga), 2014-2016, Daga I. C. 167b (PE. FCEFyN-UNC). 


\section{Clase ZygnematophyceAe \\ Actinotaenium capax var. minus (Schmidle) Teiling \\ ex Ruzicka \& Pouzar 1978 (Fig. 5C)}

Célula con una constricción poco marcada, cada hemicélula elipsoidal a casi esférica. Cloroplasto parietal con 3 a 5 bandas longitudinales y un pirenoide por hemicélula, estrellado en vista apical. Pared celular con gránulos. Longitud celular $45 \mu \mathrm{m}$; ancho celular $40 \mu \mathrm{m}$; istmo $17 \mu \mathrm{m}$.

- Hábitat y distribución: planctónica. Sin datos en Argentina (Tell, 1985).

- Material examinado: Argentina. Prov. Córdoba, Dptos. Punilla y Colón (río San Antonio y arroyos Colanchanga y Los Hornillos), 2014-2016, Daga I. C. 156, 160, 167b (PE. FCEFyN-UNC).

\section{Closterium navicula (Brébisson) Lütkemüller 1905 (Fig. 5G)}

Células de forma naviculoide, el contorno de una célula se asemeja a un bote. Vacuolas terminales de gran tamaño. Pirenoides 1 a 3 por hemicélula (no visibles en la fotografía). Célula: largo 60-90 $\mu \mathrm{m}$; ancho 20-25 $\mu \mathrm{m}$.

- Hábitat y distribución: planctónica. Sin datos en Argentina (Tell, 1985).

- Material examinado: Argentina. Prov. Córdoba, Dpto. Punilla (arroyo Las Mojarras), 2014-2016, Daga I. C. 162 (PE. FCEFyN-UNC).

\section{Cosmarium abbreviatum Raciborski 1885 (Fig. 5D)}

Célula de talla pequeña, cuerpo celular de menor tamaño en parte superior, márgenes laterales más largos. Longitud celular de $28 \mu \mathrm{m}$; ancho celular de $20 \mu \mathrm{m}$; istmo de $8 \mu \mathrm{m}$.

- Hábitat y distribución: planctónica. En Argentina citada para Entre Ríos (Tell, 1985).

- Material examinado: Argentina. Prov. Córdoba, Dpto. Punilla (rio Cosquín y San Antonio), 2014-2016, Daga I. C. 147b, 156b, 188 (PE. FCEFyN-UNC).

\section{Cosmarium formosulum Hoffman 1888 (Fig. 5E)}

Hemicélulas trapezoidales con márgenes basales ampliamente redondeados. Hemicélulas con 6 o 7 ondulaciones, ápice ligeramente ondulado (5 ondas). Vista apical elíptica. Longitud celular de 36-40 $\mu \mathrm{m}$; ancho celular de $28-32 \mu \mathrm{m}$; istmo de 8-12 $\mu \mathrm{m}$.

- Hábitat y distribución: planctónica. En Argentina citada para Antártida, Tierra del Fuego (Tell, 1985).

- Material examinado: Argentina. Prov. Córdoba, Dptos. Punilla y Colón (río San Antonio y arroyos Las Mojarras y Los Hornillos), 2014-2016, Daga I. C. 156, 167b, 173, 188, 191(PE. FCEFyN-UNC). 

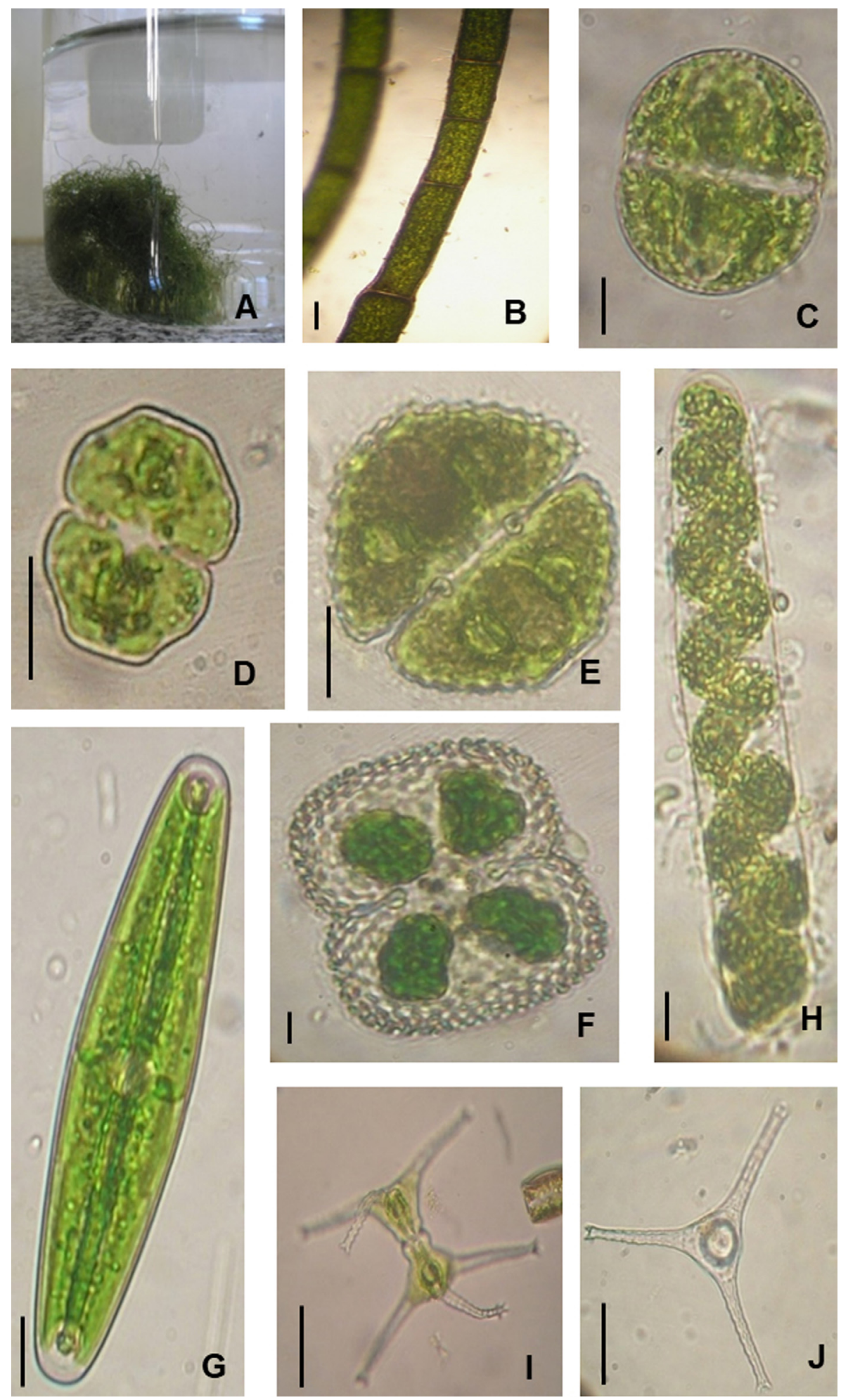

Fig. 5. A-B) Chaetomorpha herbipolensis. C) Actinotaenium capax var. minus. D) C. abbreviatum. E) Cosmarium formosulum. F) C. quadrum. G) Closterium navicula. H) Spirotaenia condensata. I-J) Staurastrum longipes.

Escala: $\mathrm{A}=1 \mathrm{~cm} ; \mathrm{B}=100 \mu \mathrm{m} ; \mathrm{C}, \mathrm{D}, \mathrm{E}, \mathrm{F}, \mathrm{G}, \mathrm{H}, \mathrm{I}, \mathrm{J}=10 \mu \mathrm{m}$.

Fig. 5. A-B) Chaetomorpha herbipolensis. C) Actinotaenium capax var. minus. D) C. abbreviatum. E) Cosmarium formosulum. F) C. quadrum. G) Closterium navicula. H) Spirotaenia condensata. I-J) Staurastrum longipes.

Scale: $A=1 \mathrm{~cm} ; \mathrm{B}=100 \mu \mathrm{m} ; \mathrm{C}, \mathrm{D}, \mathrm{E}, \mathrm{F}, \mathrm{G}, \mathrm{H}, \mathrm{I}, \mathrm{J}=10 \mu \mathrm{m}$. 
Cosmarium quadrum P. Lundell 1871 (Fig. 5F)

Hemicélulas rectangulares con esquinas redondeadas, pared ornamentada con granulaciones. Célula: largo de 50- $90 \mu \mathrm{m}$; ancho de 40- $80 \mu \mathrm{m}$.

- Hábitat y distribución: planctónica. Sin datos en Argentina (Tell, 1985).

- Material examinado: Argentina. Prov. Córdoba, Dpto. Punilla (río San Antonio), 2014-2016, Daga I. C. 156 (PE. FCEFyN-UNC).

\section{Spirotaenia condensata Brébisson 1848 (Fig. 5H)}

Células cilíndricas, 6 veces más largas que anchas con extremos redondeados. Cloroplasto parietal espiralado con numerosos pirenoides. Largo celular 100-120 $\mu \mathrm{m}$; ancho celular 20-22 $\mu \mathrm{m}$.

- Hábitat y distribución: planctónica, de ambientes mesotróficos a eutróficos (Margalef, 1983).

- Distribución: cosmopolita. En Argentina citada para Buenos Aires, Tierra del Fuego (Tell, 1985).

- Material examinado: Argentina. Prov. Córdoba, Dpto. Punilla (río San Antonio), 2014-2016, Daga I. C. 165 (PE. FCEFyN-UNC).

\section{Staurastrum longipes (Nordstedt) Teiling 1946 (Fig. 5I y5J)}

Organismos unicelulares, solitarios. Hemicélulas poligonales en vista polar, ápices prolongándose en apéndices en forma de brazos, 3 por hemicélula. Cloroplasto con un pirenoide. Longitud celular 68-74 $\mu \mathrm{m}$; ancho celular 25-28 $\mu \mathrm{m}$; ancho celular con procesos $97 \mu \mathrm{m}$; istmo $12 \mu \mathrm{m}$.

- Hábitat y distribución: planctónica. En Argentina citada para Corrientes (Tell, 1985).

- Material examinado: Argentina. Prov. Córdoba, Dpto. Punilla (río San Antonio y arroyo Las Mojarras), 2014-2016, Daga I. C. 156, 157b (PE. FCEFyN-UNC).

\section{DISCUSIÓN Y CONCLUSIONES}

Se registraron un total de 66 taxones de los cuales 22 son nuevas citas para la provincia de Córdoba. La mayor riqueza de especies ocurrió en primavera, condiciones como el aumento de temperatura y del fotoperíodo, acompañado con una disminución del caudal y la turbulencia debido a las bajas precipitaciones, favorecen el estancamiento del agua generando un ambiente apropiado para su desarrollo (de Ruyter van Steveninck et al., 1992; Luque y Martínez de Fabricius, 2003, 2005).

Las Chlorophyceae aportaron el mayor número de especies, su alta variabilidad morfométrica permite que se desarrollan en diversos hábitats (Happey-Wood, 1988) y especies como Monoraphidium griffithii; Actinastrum hantzschii, Monactinus simplex, Nephrocytium agardhianum, Pediastrum boryanum var. boryanum, $P$ simplex, $P$. duplex y Scenedesmus dimorphus (=Tetradesmus dimorphus) son indicadoras de estado eutró- 
fico (Huber-Pestalozzi, 1983; Comas Gonzaléz, 1996; Pinilla, 2000; Reynods, 2002; Phinyo et al., 2017).

De los seis cuerpos de agua estudiados en el Río San Antonio se identificaron el $77 \%$ de los taxones, una particularidad que ocurrió en este cuerpo de agua fue presentar una alta biomasa de macrófitas a fines de verano y comienzo de otoño. Estudios realizados en ríos de Brasil señalan que las macrófitas pueden crear las condiciones ideales de iluminación para el desarrollo de numerosas especies de algas verdes y además aportar nutrientes provenientes del envejecimiento y muerte de estos organismos (Felisberto y Rodrigues, 2005; Bortolini et al., 2010).

Las densidades celulares en general son mayores a las observadas para otros ríos de Córdoba (Luque y Martínez de Fabricius, 2003). El mayor aporte corresponde a Phacotus lenticularis, especie típica de ambientes alcalinos, un aumento en el $\mathrm{CO}_{3} \mathrm{Ca}$ estimula su desarrollo (Shlegel et al., 1998; Gruenert y Raeder, 2014). Su presencia en el río Cosquín y el arroyo Colanchanga nos indica la presencia de aguas con carbonato de calcio debido a la naturaleza del suelo (Gaiero, 1988). Es importante mencionar que el aumento de Phacotus puede deberse también a un posible incremento de materia orgánica producto de la intensa urbanización que han experimentado las márgenes de estos dos cuerpos de agua en los últimos años. La menor riqueza y abundancia de algas verdes ocurrió en el arroyo Los Chorrillos. Florísticamente, el mencionado arroyo presentó similitudes con el embalse San Roque, donde los organismos dominantes fueron los dinoflagelados, especies planctónicas generalmente de ambientes lénticos, su abundancia en el arroyo Los Chorrillos puede ser atribuida a la acción del viento (Daga et al., 2020).

Las especies filamentosas como Cladophora glomerata y Chaetomorpha herbipolensis fueron evidentes durante los meses de baja temperatura. Son organismos que viven fijos al sustrato del cual se desprenden debido a la acción de la velocidad de corriente y pasan a formar el parte del plancton (Luque y Martínez de Fabricius, 2003; Biasotti et al., 2014; Galea et al., 2014).

Considerando lo anteriormente expuesto se puede concluir:

- La comunidad de algas verdes presentó mayor desarrollo en las estaciones cálidas donde el aumento de la temperatura y el fotoperíodo son favorables para su desarrollo.

- Un aumento en la biomasa de Phacotus en el río Cosquín y el arroyo Colanchanga nos sugieren un posible incremento de materia orgánica como producto de la creciente urbanización.

- De los 66 taxones identificados 14 fueron citados en la bibliografía como indicadoras de estado eutrófico.

- Con este trabajo se incrementa el conocimiento de la riqueza de algas verdes para ríos y arroyos de la provincia de Córdoba. 


\section{BIBLIOGRAFÍA}

Bazán, G., Almeyda, D., Olivera, L. y Oriani, B. (2014). Biodiversidad fitoplanctónica en tres lagunas someras de la provincia La Pampa, Argentina. Biología Acuática 30: 47-57.

Biasotti, A. E., Álvarez, S. B., Bazán, G. I. y Martínez de Fabricius, A. L. (2014). Variación estacional de la comunidad microfitoplanctónica del curso medio del Río Colorado (La Pampa-Argentina). Biología Acuática 30: 249-258.

Bojorge-García, M. G. y Cantoral Uriza, E. A. (2016). La importancia ecológica de las algas en los ríos. Hidrobiológica 26 (1): 1-8.

Bortolini, J. C., Biolo, S., Bueno, N. C., Godinho, L. R. y Pott, V. J. (2010). Chlorococcales sensu lato (Chlorophyceae) em tanques de depuração de efluente de origem bovina no Mato Grosso do Sul, Brasil. Iheringia, Serie Botanica 65 (1): 63-74.

Brettum, P. y Andersen, T. (2005). The use of phytoplankton as indicators of water quality. NIVA Report.

Capitanelli, R. G. (1979). Clima. En: Geografía física de Córdoba (J. B. Vásquez, J, B., Miatello, R. A., y Roqué, M. E.) Ed. Boldt. Córdoba, Argentina, pp. 45-138.

Colladon, L. (2018). Anuario pluviométrico 2012/13-2016/17, cuenca del Río San Antonio. Informe interno del CIRSA, Instituto Nacional del Agua.

Comas González, A. (1996). Las Chlorococcales dulciacuícolas de Cuba. J. Cramer Ed. Berlin. Stuttgart. Biblioteca Phycologica.

Daga, I. C., Fernández Belmonte, M. C. y Reyna, S. M. (2020). Composición algal y bioindicadores de calidad de agua. Caso de estudio: embalse San Roque, Córdoba. Argentina. Cuadernos del Curiham 26: 1-11.

Dasso, C. M., Piovano, E. I., Pasquini, A. I., Córdoba, F. E., Lecomte, K. I., Guerra, L. y Campodónico, V. A. (2014). Recursos hídricos superficiales. Memorias XIX Congreso Geológico Argentino - Córdoba.

de Ruyter van Steveninck, E. D., Admiraal, W., Breebaart, L., Tubbing, G. M. J. y Zanten, B. van. (1992). Plankton in the river Rhine: structural and functional changes observed during downstream transport. Fournal of Plankton Research 14: 1351-1368.

Esho, R. T. y Benson-Evans, K. (1984). Algal studies of the River Ely, South Wales, U. K. I. Phytoplankton. Nova Hedwigia 40: 347-368.

Ettl, H. (1983). Chlorophyta I. Phytomonadina. En: H. Ettl, J. Gerloff, H. Heynig and D. Mollenhauer eds. Süßwasserflora von Mitteleuropa 9. Gustav Fischer Verlag, Stuttgart.

Felisberto, S. A. y Rodrigues, L. (2005). Influência do gradiente longitudinal (riobarragem) na similaridade das comunidades de desmídias perifiticas. Revista Brasileira de Botânica 28 (2): 241-254.

Gaiero, D. (1998). Hidrogeoquímica de un sistema de la región semiárida: el Río Suquía, Córdoba, 2. Transporte de sólidos disueltos, meteorización y consumo de $\mathrm{CO}_{2}$. Revista de la Asociación Geológica Argentina 53 (3): 337:347.

Galea, M. J., Bazán, G. I., Álvarez, S. B. y Martínez De Fabricius, A. L. (2014). Estudio del fitoplancton aguas arriba y aguas abajo del Dique Embalse Casa de Piedra, río Colorado (La Pampa, Argentina). Biología Acuática 30: 287-300. 
Gruenert, U. y Raeder, U. (2014). Growth responses of the calcite-loricated freshwater phytoflagellate Phacotus lenticularis (Chlorophyta) to the $\mathrm{CaCO}_{3}$ saturation state and meteorological changes. Fournal of Plankton Research 36 (3): 630-640.

Guiry, M. D. y Guiry, G. M. (2021). AlgaeBase. World-wide electronic publication, National University of Ireland, Galway. Disponible en: http://www.algaebase.org Happey-Wood, C. M. (1988). Ecology of freshwater planktonic green algae. En: Sandgren, C. D. (ed.), Growth and Reproductive strategies of freshwater phytoplankton, p. 175- 226. Cambridge University Press, New York.

Harris, G. P. (1986). Phytoplankton ecology, structure, function and fluctuation Chapman and Hall, Londres.

Huber-Pestalozzi, G. (1983). Das Phytoplankton des Süsswassers, Chlorophyceae. Ordnung Chlorococcales. En: Thienemann A. (editor) Die Binnengewasser, 16 (7), E. Schweizerbart'sche Verlagsbuchhandlung, Stuttgart.

Komárek, J. y Fott, B. (1983). Chlorophyceae Chlorococcales. Das phytoplanktom des Süsswassers, systematic u biologie. In: Thienemann, A. (ed.), Die Binnenngewässer 16, 7. E. Schweizerbart'sche Verlagbuchhandlung, Stuttgart.

Luque, M. E. y Martinez de Fabricius, A. L. (2000). Ficoflora fitoplanctónica y epilítica del río Piedra Blanca. Boletín de la Sociedad Argentina de Botánica 35 (1-2): 21-32.

Luque, M. E. y Martínez de Fabricius, A. L. (2003). Distribución temporal del fitoplancton y epiliton en el río Piedra Blanca (Córdoba, Argentina). Limnetica 22 (3-4): 19-34.

Luque, M. E. y Martínez de Fabricius, A. L. (2005). Algas fitoplanctónicas del Río Piedra Blanca (Córdoba, Argentina) y su relación con los factores ambientales. Lilloa 42 (1-2): 69-79.

Margalef, R. (1983). Limnología. Barcelo na: Ediciones Omega, S. A.

Moraña, L. (2013). FitopIancton de ríos y arroyos de Ia AIta Cuenca deI Río Bermejo, Argentina. Lhawet/ Nuestro entorno. PubIicación deI Instituto de EcoIogía y Ambiente Humano VoIumen II Número I.

Novelo, E. (2012). Chlorophyta. Flora del Valle de Tehuacán Cuicatlán. Instituto de Biología, UNAM, México, D. F. Fascículo 94:1-86.

Phinyo, K., Pekkoh, J. y Peerapornpisal, Y. (2017). Distribution and ecological habitat of Scenedesmus and related genera in some freshwater resources of Northern and North-Eastern Thailand. Biodiversitas 18: 1092-1099. 10.13057/bio$\mathrm{div} / \mathrm{d} 180329$.

Pinilla, G. A. (2000). Indicadores biológicos en ecosistemas acuáticos continentales de Colombia. Bogotá. Fundación Universidad de Bogotá Jorge Tadeo Lozano.

Prescott, G. W. (1982). Algae of the Western Great Lakes Area. W.M.C. Brown Co. Pub., Dubuque.

Reynolds, C. S., Vera, H., Kruk, C., Naselli-Flores, L. y Melo, S. (2002). Towards a functional classification of the freshwater phytoplankton. Fournal of Plankton Research 24 (5): 417-428.

Salmaso, N., Naselli-Flores, L., Padisak, J. (2015). Functional classifications and their application in phytoplankton ecology. Freshwater Biology 60 (4), 603-619. 
Schlegel, I., Koschel, R. y Krienitz, L. (1998). On the occurrence of Phacotus lenticularis (Chlorophyta) in lakes of different trophic state. Hydrobiologia 69: 353361.

Stoyneva, M. P. y Gärtner, G. (2004). Taxonomic and ecological notes to the list of green algal species from Bulgarian thermomineral waters. Berichte des Naturwissenschaftich-Medizinischen Vereins in Innsbruck 91:67-89.

Taboada, M. A. y Tracanna, B. (2013). Ficoflora y Calidad del Agua del Arroyo Mista (Tucumán-Argentina). Editorial Académica Española, Barcelona.

Tell, G. (1979). Chlorophyceae d'eau douce rares et nouvelles de la Republique Argentine. Revue Algologique, Nouvelle Serie 14: 39-48.

Tell, G. (1985). Catálogo de las Algas de Agua Dulce de la República Argentina. Bibliotheca Phycologica 70. Vaduz: J. Cramer.

Vázquez, J., Miatello, R. y Roque, M. (1979). Geografía Física de la Provincia de Córdoba. Ed. Boldt. Argentina.

Yulu, T., Yuan, J., Qi L., Dingxue, X., Yang, L. y Jinxi, S. (2021). The impacts of local and regional factors on the phytoplankton community dynamics in a temperate river, northern China, Ecological Indicators V. 123.

Zalocar de Domitrovic, Y. (1999). Estructura y dinámica del fitoplancton en la cuenca del eje potámico Paraguay-Paraná (Argentina). (Tesis doctoral), Universidad Nacional de Córdoba, Argentina. 